Keywords: biochemical recurrence; HR-MAS MRS; magnetic resonance spectroscopy; metabolomics; prognostic biomarkers; prostate cancer; spermine; citrate

\title{
Ex vivo metabolic fingerprinting identifies biomarkers predictive of prostate cancer recurrence following radical prostatectomy
}

Peder R Braadland ${ }^{1,2}$, Guro Giskeødegård ${ }^{3}$, Elise Sandsmark ${ }^{3}$, Helena Bertilsson ${ }^{4,5}$, Leslie R Euceda ${ }^{3}$, Ailin F Hansen ${ }^{3}$, Ingrid J Guldvik ${ }^{1}$, Kirsten M Selnæs ${ }^{3}$, Helene H Grytli ${ }^{1}$, Betina Katz ${ }^{6}$, Aud Svindland ${ }^{2,6}$, Tone F Bathen ${ }^{3}$, Lars M Eri ${ }^{2,7}$, Ståle Nygård ${ }^{8}$, Viktor Berge ${ }^{7}$, Kristin A Taskén*,1,2 and May-Britt Tessem ${ }^{\star, 3}$

${ }^{1}$ Department of Tumor Biology, Institute for Cancer Research, Oslo University Hospital, PO Box 4953 Nydalen, Oslo 0424, Norway; ${ }^{2}$ Institute of Clinical Medicine, Faculty of Medicine, University of Oslo, Oslo 0313, Norway; ${ }^{3}$ Department of Circulation and Medical Imaging, Faculty of Medicine and Health Sciences, NTNU - Norwegian University of Science and Technology, Postboks 8905, Trondheim 7491, Norway; ${ }^{4}$ St Olavs Hospital, Trondheim University Hospital, Trondheim 7030, Norway; ${ }^{5}$ Department of Cancer Research and Molecular Medicine, Faculty of Medicine, NTNU - Norwegian University of Science and Technology, Trondheim 7491, Norway; ${ }^{6}$ Department of Pathology, Oslo University Hospital, Oslo 0424, Norway; ${ }^{7}$ Department of Urology, Oslo University Hospital, Oslo 0424, Norway and ${ }^{8}$ Bioinformatics Core Facility, Institute for Medical Informatics, Oslo University Hospital, Oslo 0424, Norway

Background: Robust biomarkers that identify prostate cancer patients with high risk of recurrence will improve personalised cancer care. In this study, we investigated whether tissue metabolites detectable by high-resolution magic angle spinning magnetic resonance spectroscopy (HR-MAS MRS) were associated with recurrence following radical prostatectomy.

Methods: We performed a retrospective ex vivo study using HR-MAS MRS on tissue samples from 110 radical prostatectomy specimens obtained from three different Norwegian cohorts collected between 2002 and 2010. At the time of analysis, 50 patients had experienced prostate cancer recurrence. Associations between metabolites, clinicopathological variables, and recurrencefree survival were evaluated using Cox proportional hazards regression modelling, Kaplan-Meier survival analyses and concordance index (C-index).

Results: High intratumoural spermine and citrate concentrations were associated with longer recurrence-free survival, whereas high (total-choline + creatine)/spermine (tChoCre/Spm) and higher (total-choline + creatine)/citrate (tChoCre/Cit) ratios were associated with shorter time to recurrence. Spermine concentration and tChoCre/Spm were independently associated with recurrence in multivariate Cox proportional hazards modelling after adjusting for clinically relevant risk factors (C-index: 0.769; HR: 0.72; $P=0.016$ and C-index: 0.765; HR: 1.43; $P=0.014$, respectively).

Conclusions: Spermine concentration and tChoCre/Spm ratio in prostatectomy specimens were independent prognostic markers of recurrence. These metabolites can be noninvasively measured in vivo and may thus offer predictive value to establish preoperative risk assessment nomograms.

Despite the curative intent of radical prostatectomy for localised prostate cancer, $15-30 \%$ of men who undergo resection develop biochemical recurrence (BCR) (Pound et al, 1999; Ward et al,
2003). Although BCR does not necessarily lead to lethal disease, $34 \%$ of men who experience BCR progress to develop metastases within 8 years following radical prostatectomy (Pound et al, 1999).

*Correspondence: Professor KA Taskén; E-mail: k.a.tasken@medisin.uio.no or Dr M-B Tessem; E-mail: may-britt.tessem@ntnu.no

Received 30 June 2017; revised 18 August 2017; accepted 1 September 2017; published online 3 October 2017

(C) 2017 Cancer Research UK. All rights reserved 0007 - 0920/17 
Novel markers capable of predicting recurrence are thus needed to identify patients eligible for treatment and active surveillance.

Current decision-making nomograms are commonly based on clinical staging, serum prostate-specific antigen (PSA), and histological findings on tissue biopsies (Canfield et al, 2014). Unfortunately, limitations to these parameters used in clinical practice lead to overdiagnosis of men with indolent disease, and concurrently aggressive cancers are missed. Several promising molecular tests for predicting BCR following radical prostatectomy are emerging, such as the genetic signature-based Prolaris assay (Bishoff et al, 2014), OncotypeDX Genomic Prostate Score (Cullen et al, 2015), and the Decipher genomic classifier (Erho et al, 2013). These tests require, however, invasive sampling by needle biopsies of significant cancer foci, which is challenging owing to the multifocality and heterogeneity of prostate cancer (Canfield et al, 2014), and do not have the noninvasive potential of whole-prostate characterisation, such as magnetic resonance (MR) imaging modalities that are appealing alternatives as prognostic tools.

Magnetic resonance spectroscopy (MRS) enables concurrent identification and quantification of several metabolites, which collectively constitute the metabolic fingerprint of a tissue sample (Kumar et al, 2016). In 2010, a retrospective ex vivo study displayed the potential of distinguishing non-recurrent from recurrent prostate cancers in a small cohort of patients after RP using metabolite profiles obtained by ex vivo high-resolution magic angle spinning MRS (HR-MAS MRS) (Maxeiner et al, 2010). The paper received attention as the findings suggested a potential utility of MRS-based technology preoperatively (Sciarra, 2010). The potential clinical utility of this was further demonstrated with data from our group showing correlations between metabolites identified ex vivo and in vivo using HR-MAS MRS and MRS imaging (MRSI), respectively (Selnaes et al, 2013). We also showed that low concentrations of spermine and citrate as well as a high (choline + creatine + polyamines)/citrate ratio were associated with high Gleason score using HR-MAS MRS (Giskeodegard et al, 2013). Recently, the number of polyamine-free voxels from in vivo MRSI) was reported to be positively associated with BCR following radical prostatectomy (Zakian et al, 2016).

In vivo MRSI has lower sensitivity and resolution than ex vivo MRS, and metabolites visible in in vivo prostate MRSI are usually limited to citrate, total-choline, creatine, and polyamines. The low resolution causes overlapping peaks, which hampers quantification of individual metabolites, and the lack of robust internal standards has warranted calculations of metabolite ratios. However, new developments in MRSI allow for absolute metabolite quantification (Weis et al, 2016), and faster and more automated protocols. Furthermore, implementation of higher magnetic field strengths and new pulse sequences can increase sensitivity (Steinseifer et al, 2015; Lagemaat et al, 2016). Thus, MRSI has the potential to play a future role in clinical practice to improve precision medicine.

In the current study, we identified prognostic metabolites predicting recurrence using ex vivo HR-MAS MRS on tissue samples from 110 patients treated with radical prostatectomy. We show that metabolic profiling provides prognostic information independently of clinicopathological parameters, and discuss the possibility for in vivo use.

\section{MATERIALS AND METHODS}

Patient selection. Patients $(n=136)$ radically operated for prostate cancer between 2002 and 2010 at Oslo University Hospital (Oslo, Norway) (OUS cohort, $n=55$ ) or at St Olavs Hospital (Trondheim, Norway) (NTNU1 cohort, $n=39$ and NTNU2 cohort, $n=42$ ) were retrospectively included in this study. Patients were excluded owing to the lack of follow-up data $(n=5)$, lack of available tumour tissue $(n=5)$, unsatisfactory HR-MAS MRS spectral quality $(n=4)$, and due to administration of adjuvant and/ or neoadjuvant therapy $(n=12)$, leaving a total of 110 patients eligible for analysis. Recurrent prostate cancer was defined as BCR (PSA $\geqslant 0.2 \mathrm{ng} \mathrm{ml}^{-1}$ with a subsequent rise). If PSA measurements were missing ( $n=2$ patients), time of recurrence was set to onset of salvage radiation therapy or salvage androgen deprivation therapy.

Ethical approval of studies and informed consent. The studies were approved by the Regional Committee for Medical and Health Research Ethics (REC) (2009/1028 and 2013/1713 REC South-East and 2009/1161 and 010-04 REC Central), and informed written consent was obtained from all included patients.

Sample collection. Samples were kindly provided by the 'Registry and Biobank for Urological Diseases' (OUS cohort, $n=55$ ), MR Biobank (NTNU1 cohort, $n=39$ ), and Biobank1, St Olavs Hospital (NTNU2 cohort, $n=104$ ). The tissue samples from the OUS cohort were collected at the Oslo University Hospital immediately after prostatectomy by excising and fresh-freezing tissue samples from areas identified as tumour by a pathologist. The NTNU1 cohort (MR Biobank) consists of tissue biopsies from St Olavs University Hospital taken within $\sim 1-2$ min after surgical removal of the prostate and immediately stored in liquid nitrogen (Hansen et al, 2016; Sandsmark et al, 2017). The biopsies ( 10 mg) were aimed at cancer areas previously detected by transrectal ultrasound guided biopsies with more than $1 \mathrm{~mm}$ tumour. The NTNU2 cohort (Biobank 1) was collected as 2 -mm fresh-frozen prostate slices from the middle of the prostate, and tissue cores $(\sim 10 \mathrm{mg})$ were extracted based on clinical histopathology on the two adjacent tissue slices (Bertilsson et al, 2011). Further protocols for sample collection following radical prostatectomy are described in Supplementary Materials and methods.

Ex vivo HR-MAS MRS. Ex vivo HR-MAS MRS metabolic fingerprinting was performed as described previously (Giskeodegard et al, 2013) using frozen, intact tissue samples (mean weight $12.5 \mathrm{mg}$, range $3.00-21.9 \mathrm{mg}$ ) on a Bruker Avance DRX600 (14.1T) Spectrometer (Bruker Biospin, Rheinstetten, Germany) equipped with a ${ }^{1} \mathrm{H} /{ }^{13} \mathrm{C}$ HR-MAS probe. Absolute quantification of the MR spectra was performed using LCModel (Provencher, 1993; Giskeodegard et al, 2013; Hansen et al, 2016) and reported in $\mathrm{nmolmg}^{-1}$ wet weight. Total choline was calculated as the sum of choline, phosphocholine, and glycerophosphocholine (GPC).

Pathology. Experimental procedures for pathological examination of the OUS cohort (AS and BK) following HR-MAS MRS spectral acquisition are described in Supplementary Materials and methods. The pathological procedures on paraffin sections (NTNU1 cohort) and cryosections (NTNU2 cohort) are described in Hansen et al (2016), Sandsmark et al (2017), and Giskeodegard et al (2013), respectively. In all three cohorts, percentages of cancer, benign (glandular) epithelium, and stromal tissue were reported for each sample.

Statistics. Linear mixed modelling (LMM) was applied to test each metabolite concentration or ratio as a function of recurrence status at 5 years while correcting for multiple samples per patient ( $\mathrm{R}$ version 3.0.3, NLME package). Metabolite concentrations and ratios were Box-Cox-transformed before analysis, and the Benjamini-Hochberg method (Benjamini and Hochberg, 1995) was used to adjust for multiple testing, giving false discovery ratecorrected $P$-values ( $Q$-values, $Q)$.

Recurrence was used as the end point in survival analyses, with time to event calculated from date of radical prostatectomy to onset of recurrence. Cox proportional hazards modelling ( $\mathrm{R}$ version 3.0.3, survival package) was performed on univariate 
and multivariate models to evaluate the association of metabolites with recurrence. Clinicopathological variables were found to be significantly associated with recurrence in univariate models, and were included as covariates in multivariate models. In all analyses, Gleason scores were categorised according to the ISUP (International Society of Urological Pathology) Grade Group System (Gordetsky and Epstein, 2016) after directly converting the reported Gleason scores as follows: Gleason score 6, 7a, 7b, 8, and 9 corresponds to Grade Group 1, 2, 3, 4, and 5, respectively. The predictive accuracies of the models were tested with the Harrell's concordance index (C-index) (Harrell et al, 1982). To compare models with different number of covariates and investigate overfitting, leave-one-out cross-validation of C-indexes (LOOCV C-index) was performed. Leave-one-out cross-validation of C-index was performed in $\mathrm{R}$ v.3.03 as follows: For each individual $i$, we first fit a Cox model to the survival data, leaving $i$ out in the estimation. The resulting coefficient estimates, $\beta_{-i}$, were then used together with individual $i$ 's covariate values $x_{i}$, to obtain the linear predictor $\eta_{i}=\beta_{-i} x_{i}$ for each individual $i$. This was done successively for each individual, thus obtaining a vector of linear predictors $\eta=\eta_{1}, \eta_{2}, \ldots, \eta_{n}$, where $n$ is the number of individuals. This vector $\eta$ was then used as a single covariate in the Cox proportional hazard model to calculate the $\mathrm{C}$-index, which we refer to as the LOOCV C-index.

Selection of metabolites to be modelled in survival analyses was based on $Q$-values from LMM and the possibility of measuring by in vivo MRSI in its current technological state. The metabolite concentrations and ratios were $\log _{2}$-transformed before Cox proportional hazards modelling to obtain scale-independent hazard ratios. For all survival analyses, one sample was randomly selected for inclusion where more than one tumour sample was present for a patient (applicable only for the NTNU2 cohort). To validate the Cox proportional hazard models, 1000 computations with random selections of samples from patients with multiple available samples were run.

Decision curve analyses were performed in $\mathrm{R}$ (version 3.0.3, DecisionCurve package) to evaluate the multivariate models at different threshold probabilities for recurrence within the 5-year mark. Recurrence-free patients lacking 5 years of follow-up were excluded $(n=4)$. One patient lacked only 15 days to reach 5 years of follow-up, but was included nonetheless, leaving a total of 106 patients for the analyses. A basic model containing pathological variables included in the Cox proportional hazards multivariate models, a full model with spermine or (total-choline + creatine)/ spermine (tChoCre/Spm), a 'treat all' function (calculated from the basic model), and a 'treat none' function were tested. Briefly, net benefit was evaluated in the threshold probability range of $0-40 \%$, with bootstrapping $(n=500)$.

The Kaplan-Meier method was used to depict differences in recurrence-free survival among patients harbouring tumours with above or below median values of the metabolites of interest, and the Mantel-Haenszel log-rank test was used to evaluate the differences in the distributions ( $\mathrm{R}$ v.3.03, survival package). To test the validity of the results from the OUS cohort in the two other cohorts, the cutoff points yielding the lowest log rank $P$-values were used (Budczies et al, 2012).

Student's $t$-tests and exact Mantel-Haenszel linear-by-linear association tests were used to test for differences in distributions of clinicopathological demographics according to recurrence, and Mann-Whitney $U$-tests were performed to test for metabolite levels in the OUS and NTNU cohorts individually (all three tests were performed in SPSS v.21; IBM, Chicago, IL, USA). Spearman's rank correlation analyses were performed using R (R v.3.03, Hmisc package).

Partial least-squares discriminant analysis (PLS-DA) was performed in MATLAB (Mathworks, Natick, MA, USA) and PLS-toolbox (Eigenvector Research, Manson, WA, USA) to search for systematic differences in metabolite concentrations between the cohorts. PLS-DA was performed with leave-one-out cross-validation using $n=10 \%$ of the patients $(n=11)$. Permutation testing $(n=1000)$ was performed to examine the significance of the resulting model.

\section{RESULTS}

Patient characteristics. Of the 110 patients eligible for analysis, recurrence was observed for 50 patients at the time of follow-up. The median follow-up time was 2366 days for patients without recurrence and 900 days for patients with recurrence. The recurrent group had a significantly higher proportion of patients with extraprostatic extension (pT3a), seminal vesicle invasion $(\geqslant \mathrm{pT} 3 \mathrm{~b})$, and had higher Gleason grades, whereas no significant differences in age, preoperative PSA, and surgical margin status were detected. All clinicopathological characteristics of the pooled cohort are presented in Table 1.

Metabolite concentrations in non-recurrent and recurrent prostate cancers. A total of 25 metabolites were quantified from the HR-MAS MRS spectra (Figure 1 and Supplementary Table S1). The spermine concentration (the most predominant polyamine in the polyamine region; Giskeodegard et al, 2013) was significantly lower in the recurrent compared with the non-recurrent group $(P=0.001, Q=0.025)$, whereas citrate approached statistical significance towards a decrease after correcting for multiple testing $(P=0.007, Q=0.063)$. Neither choline, total choline (not shown), nor creatine were significantly different between the groups.

The tChoCre/Spm ratio was significantly higher in samples from patients who experienced recurrence within 5-years of followup $(P=0.002, Q=0.025)$. The (total choline + creatine $) /$ citrate (tChoCre/Cit) ratio approached statistical significance towards separating the groups $(P=0.011, Q=0.079)$ (Figure $1 \mathrm{~B}$ and Supplementary Table S1). Additionally, spermine and citrate concentrations were found to be highly correlated (Spearman's $\rho=0.79, P<0.001$ ) (Supplementary Figure S1).

\begin{tabular}{|c|c|c|c|}
\hline & $\begin{array}{c}\text { No recurrence } \\
(\%)\end{array}$ & $\begin{array}{l}\text { Recurrence } \\
(\%)\end{array}$ & $P$-value \\
\hline$N$ & 60 & 50 & \\
\hline $\begin{array}{l}\text { Follow-up } \\
\quad \text { Median (IQR), d }\end{array}$ & $2366(455)$ & $900(1148)$ & \\
\hline $\begin{array}{l}\text { Age } \\
\text { Mean } \pm \text { s.d. (years) }\end{array}$ & $61.7 \pm 6$ & $62.5 \pm 5$ & $0.42^{\mathrm{a}}$ \\
\hline $\begin{array}{l}\text { Preoperative PSA } \\
\text { Mean } \pm \text { s.d. }\left(n g \mathrm{ml}^{-1}\right)\end{array}$ & $9.4 \pm 5.8$ & $10.3 \pm 5.1$ & $0.44^{a}$ \\
\hline $\begin{array}{l}\text { Grade group (RP) } \\
1 \\
2 \\
3 \\
4 \\
5\end{array}$ & $\begin{array}{c}4(7) \\
39(65) \\
15(25) \\
2(3) \\
0(0)\end{array}$ & $\begin{aligned} & 5(10) \\
& 14(28) \\
& 17(34) \\
& 7(14) \\
& 7(14)\end{aligned}$ & $<0.001^{b}$ \\
\hline EPE & $9(15)$ & $31(62)$ & $<0.001^{b}$ \\
\hline SVI & $4(7)$ & $12(24)$ & $0.014^{b}$ \\
\hline PSM & $15(25)$ & $21(42)$ & $0.12^{b}$ \\
\hline PCa-specific death & 0 & $3(6)$ & \\
\hline \multicolumn{4}{|c|}{ 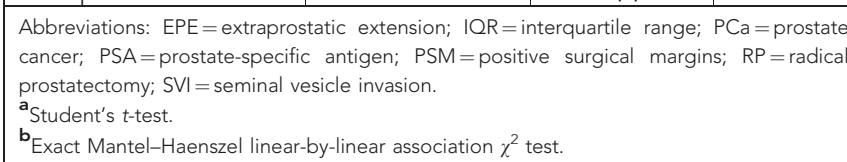 } \\
\hline
\end{tabular}



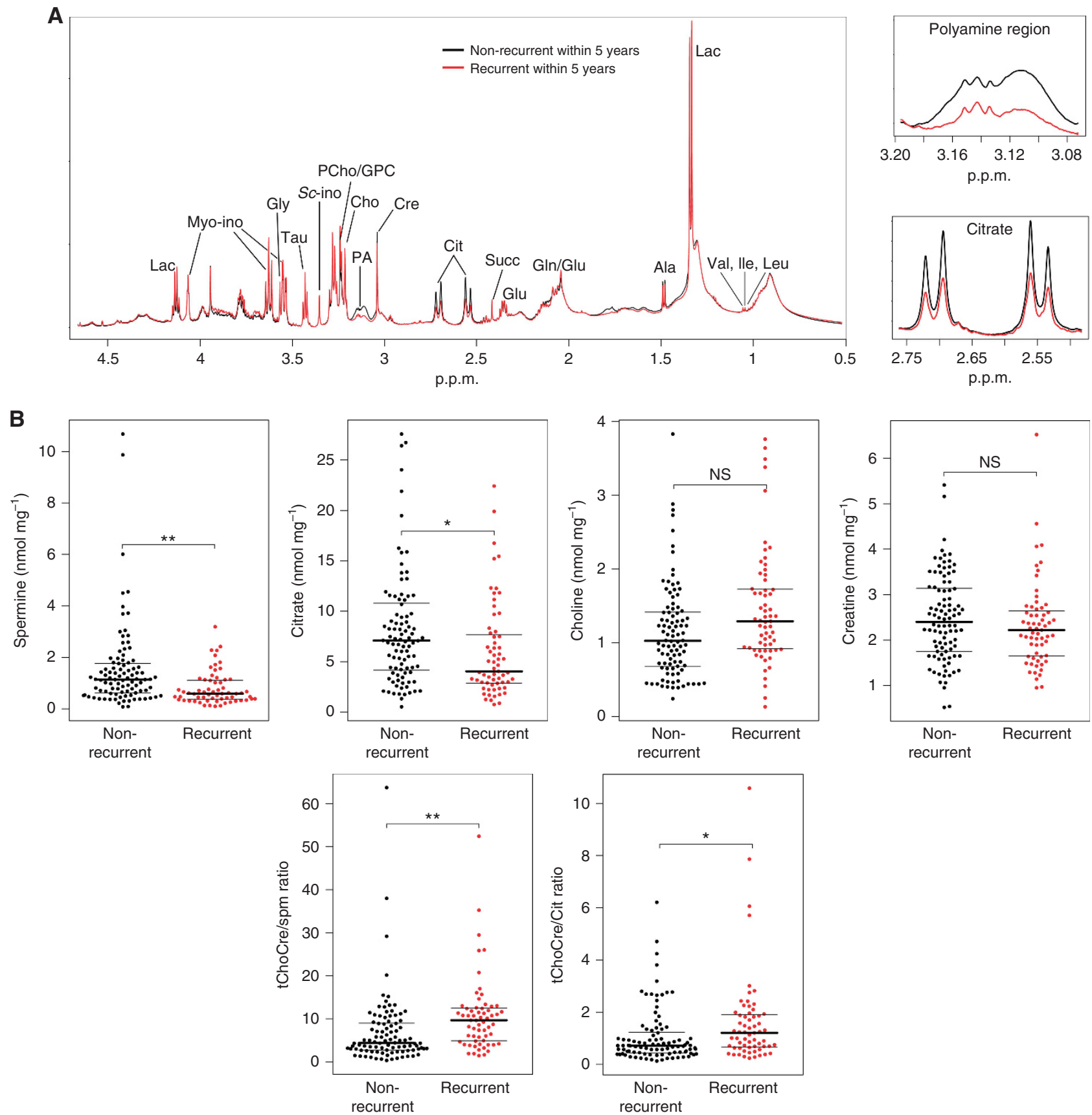

Figure 1. Metabolite levels in non-recurrent and recurrent prostate cancers at the 5-year mark. (A) Average HR-MAS MRS spectra from tumours from non-recurrent (black) and recurrent (red) prostate cancer groups in the NTNU1 cohort. Magnifications of the polyamine (containing spermine

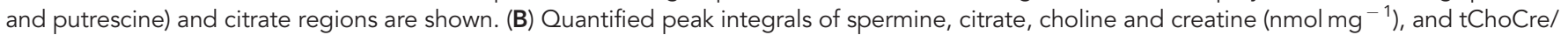
$\mathrm{Spm}$ and tChoCre/Cit ratios in the groups from all samples in all included cases from the three cohorts are shown as beeswarm plots ( $n=158)$. The thin horizontal lines make out the $25 \%$ and $75 \%$ quartiles, and the median value is shown as thick black horizontal lines. Statistical significance was calculated by LMM to account for multiple samples per patient $\left.{ }^{\star} P<0.05,{ }^{\star \star} \mathrm{Q}<0.05\right)$. Ala, alanine; Cho, choline; Cit, citrate; Cre, creatine; Gln, glutamine; Glu, glutamate; Gly, glycine; Lac, lactate; Myo-ino, myo-inositol; NS, nonsignificant; PA, polyamines; PCho/GPC, phosphocholine and glycerophosphocholine peaks; Sc-ino, scyllo-inositol; Spm, spermine; Succ, succinate; Tau, taurine; tCho, total choline.

After stratifying patients based on median metabolite or ratio levels, patients with high levels of spermine and citrate had a longer recurrence-free survival than patients with low levels of these metabolites as shown in the Kaplan-Meier plots (Figure 2). For metabolite ratios, low ratios were associated with longer recurrence-free survival.

Predictive accuracy of metabolites for prostate cancer recurrence. Candidate metabolites with translational potential for in vivo MRSI were evaluated in Cox proportional hazards univariate and multivariate modelling to investigate their association with recurrence-free survival. In univariate Cox analyses, higher spermine and citrate concentrations were associated with a decreased risk of recurrence, whereas higher choline concentration, as well as the tChoCre/Spm and tChoCre/Cit ratios, were associated with an increased risk of recurrence (Table 2). The spermine concentration and the tChoCre/Spm ratio were independently associated with recurrence-free survival in multivariate 

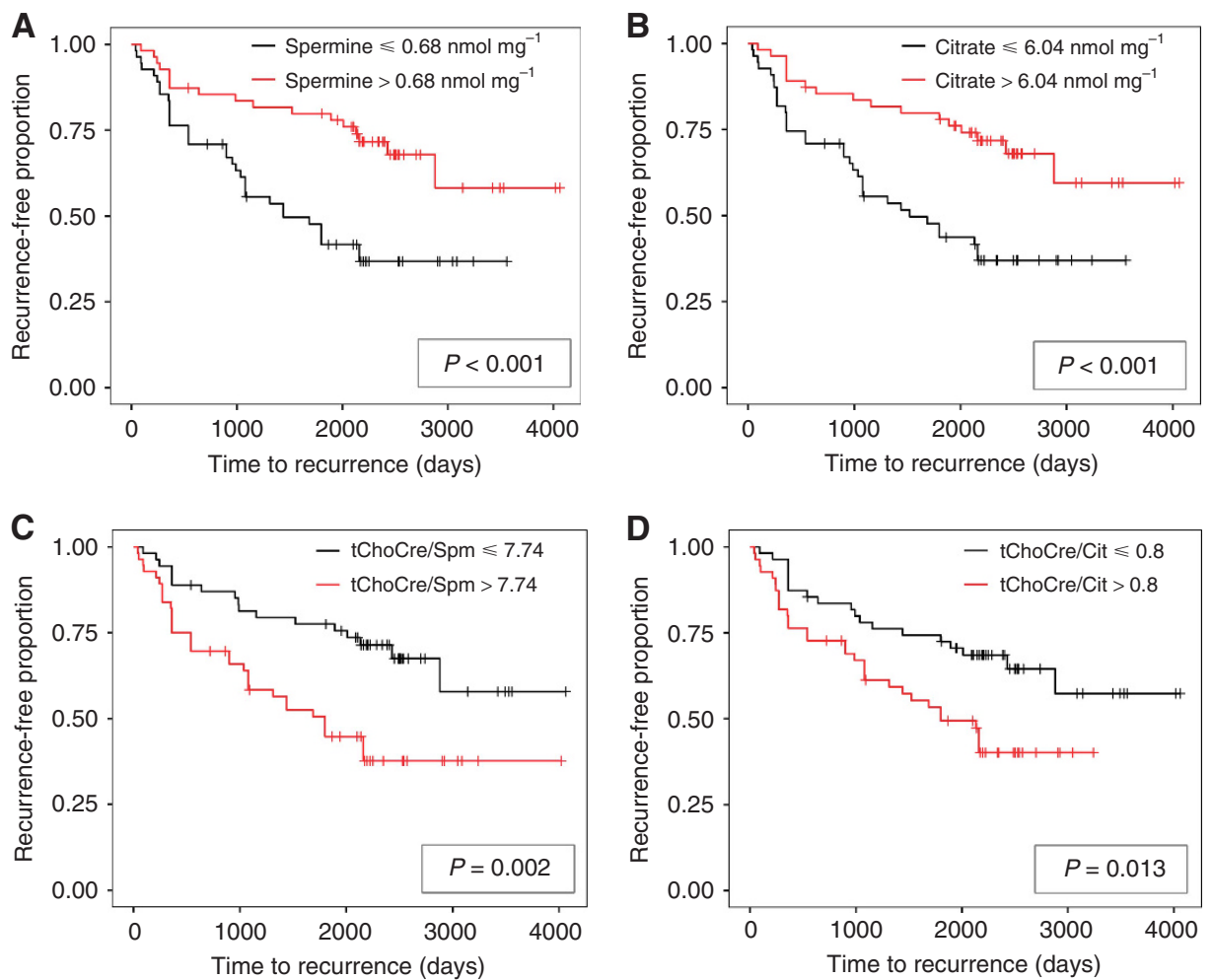

Figure 2. Kaplan-Meier curves for patients with high and low levels of metabolites and ratios. Recurrence-free proportions plotted against time to first report of recurrence for spermine (A), citrate (B), tChoCre/Spm (C), and tChoCre/Cit (D) dichotomised to above and below median concentrations. Mantel-Haenszel log-rank test was used to test for the null hypothesis of equal survival distributions. tChoCre/Cit, (totalcholine + creatine)/citrate; tChoCre/Spm, (total-choline + creatine)/spermine.

models after adjusting for variables found to be predictive of recurrence in univariate models (Grade Group, EPE, SVI) (spermine model and tChoCre/Spm model; Table 2). The calculated hazard ratios were in good compliance with the output from random sampling where more than one sample was available for a patient (Supplementary Figure S2). The hazard ratio of citrate was statistically significant in the univariate Cox analysis, suggesting high citrate levels to be associated with lower risk of recurrence. However, citrate was not statistically significant in the multivariate Cox model (Supplementary Table S2). Similarly, although not statistically significant, creatine was associated with a lower risk of recurrence, whereas choline and tChoCre/Cit were associated with an increased risk of recurrence in multivariate models.

The spermine concentration and the tChoCre/Spm ratio reached univariate C-statistics (predictive accuracy) of 0.667 and 0.660 , respectively, which were higher than that of seminal vesicle invasion, but not extraprostatic extension and Grade Group (Table 3). A basic clinicopathological model containing Grade Group (1-5), extraprostatic extension and seminal vesicle invasion reached a LOOCV C-index of 0.749. Adding spermine or tChoCre/ Spm to the basic model increased the LOOCV C-index to 0.769 and 0.765 , respectively, thus both provided additional predictive power over clinicopathological parameters alone.

Decision curve analyses visualise the net benefit of a model according to different threshold probabilities (i.e. chance of recurrence based on evaluated risk factors) at which patients or clinicians may consider performing additional prognostic tests (Vickers and Elkin, 2006). In this study, decision curve analyses revealed a positive net benefit of adding spermine to the basic clinicopathological model at decision threshold probabilities from 13 to $28 \%$ (Supplementary Figure S3). For tChoCre/Spm, the net benefit was positive in the decision threshold range $14-20 \%$.
Variability between the three pooled cohorts. As the three cohorts were pooled, we investigated the reproducibility across the cohorts. The clinicopathological characteristics of each cohort is presented in Supplementary Table S3. The histopathological composition of the tissue samples analysed is shown in Supplementary Figure S4. The distribution of cancer tissue was highest in the NTNU2 cohort (mean 62\%), which had the highest distribution of Grade Group $\geqslant 4$ samples, whereas tissue samples from the NTNU1 and OUS cohorts had less cancerous tissue (mean 38\% and 35\%, respectively), but higher stromal content (mean $40 \%$ and $57 \%$, respectively). The amount of benign tissue was nonsignificantly lower in recurrent than in non-recurrent samples from the NTNU1 cohort.

Bar charts and a PLS-DA score plot describing the metabolic differences between the three cohorts are shown in Supplementary Figure S5. The median metabolite concentrations across the cohorts showed similar trends, although the median concentrations and size of interquartile ranges displayed intercohort variance. A PLS-DA model with three latent variables showed significant differences in metabolite concentrations between the cohorts $(P<0.001)$ with an average classification accuracy of $92 \%$ (sensitivity $=91.7 \%$, specificity $=92.3 \%)$. The loadings revealed that the OUS cohort was systematically different from the other two cohorts in its levels of isoleucine, leucine, glycerophosphoethanolamine and citrate. The separation of the NTNU1 cohort was characterised by its taurine, valine, glutamate and myoinositol levels, whereas NTNU2 had differential GPC, glutamine and ethanolamine levels. The Spermine concentration did not contribute to the variance observed between the cohorts.

We tested the validity of results obtained in the OUS cohort, which was most balanced in terms of clinicopathology, in the NTNU1 and NTNU2 cohorts separately. The cutoff points for metabolite concentrations and ratios that best separated the recurrent and non-recurrent group in the OUS cohort were 
Table 2. Univariate and multivariate Cox proportional hazard ratios for metabolites and metabolite ratios and prostate cancer recurrence following radical prostatectomy

\begin{tabular}{|c|c|c|c|c|c|c|}
\hline \multirow[b]{3}{*}{ Variable } & \multicolumn{2}{|c|}{ Univariate analysis } & \multirow{2}{*}{\multicolumn{2}{|c|}{$\begin{array}{c}\text { Multivariate analysis } \\
\text { Spermine model }\end{array}$}} & \multirow{2}{*}{\multicolumn{2}{|c|}{$\begin{array}{l}\text { Multivariate analysis } \\
\text { tChoCre/Spm model }\end{array}$}} \\
\hline & \multirow[b]{2}{*}{ HR $(95 \% \mathrm{Cl})$} & \multirow[b]{2}{*}{$P$-value } & & & & \\
\hline & & & $\mathrm{HR}(95 \% \mathrm{Cl})$ & $P$-value & HR $(95 \% \mathrm{Cl})$ & $P$-value \\
\hline tChoCre/Spm $\left(\log _{2}\right)$ & $1.55(1.23-1.96)$ & $<0.001$ & & & $1.43(1.08-1.91)$ & 0.014 \\
\hline Citrate $\left(\log _{2}\right)$ & $0.67(0.51-0.86)$ & 0.002 & & & & \\
\hline Creatine $\left(\log _{2}\right)$ & $0.75(0.48-1.19)$ & 0.22 & & & & \\
\hline Age (continuous) & $1.02(0.97-1.07)$ & 0.42 & & & & \\
\hline Preoperative PSA & $1.02(0.98-1.07)$ & 0.329 & & & & \\
\hline $\begin{array}{c}\text { Grade group (RP) } \\
1 \\
2 \\
3 \\
4 \\
5\end{array}$ & $\begin{array}{c}\text { Reference } \\
0.38(0.14-1.05) \\
0.92(0.34-2.51) \\
2.12(0.67-6.71) \\
3.01(0.95-9.56)\end{array}$ & $\begin{array}{l}0.062 \\
0.87 \\
0.20 \\
0.061\end{array}$ & $\begin{array}{c}\text { Reference } \\
0.55(0.18-1.70) \\
0.83(0.28-2.44) \\
2.73(0.81-9.18) \\
2.04(0.61-6.85)\end{array}$ & $\begin{array}{l}0.30 \\
0.74 \\
0.11 \\
0.25\end{array}$ & $\begin{array}{c}\text { Reference } \\
0.53(0.17-1.65) \\
0.73(0.24-2.23) \\
2.58(0.76-8.72) \\
2.00(0.60-6.71)\end{array}$ & $\begin{array}{l}0.27 \\
0.59 \\
0.13 \\
0.26\end{array}$ \\
\hline EPE & $4.65(2.61-8.27)$ & $<0.001$ & $3.03(1.54-5.96)$ & 0.0013 & $2.98(1.51-5.89)$ & 0.0017 \\
\hline SVI & $3.39(1.76-6.54)$ & $<0.001$ & $1.02(0.46-2.72)$ & 0.95 & $1.06(0.49-2.31)$ & 0.87 \\
\hline PSM & $1.67(0.95-2.94)$ & 0.074 & & & & \\
\hline
\end{tabular}

Table 3. C-index for univariate and multivariate models including spermine and the tChoCre/Spm metabolite ratio

\begin{tabular}{|c|c|c|c|c|c|}
\hline \multicolumn{3}{|c|}{ Univariate analysis } & \multicolumn{3}{|c|}{ Multivariate analysis } \\
\hline Variable & C-index & & C-index & LOOCV C-index ${ }^{a}$ & $\Delta \mathrm{C}$-index \\
\hline Spermine $\left(\log _{2}\right)$ & 0.667 & \multirow{2}{*}{ Full models } & 0.769 & 0.769 & 0.020 \\
\hline tChoCre/Spm (log 2$)$ & 0.660 & & 0.765 & 0.765 & 0.016 \\
\hline Grade group (RP) & 0.694 & \multirow{3}{*}{ Basic model } & \multirow{3}{*}{0.749} & \multirow{3}{*}{0.749} & \\
\hline EPE & 0.697 & & & & \\
\hline SVI & 0.595 & & & & \\
\hline \multicolumn{6}{|c|}{ 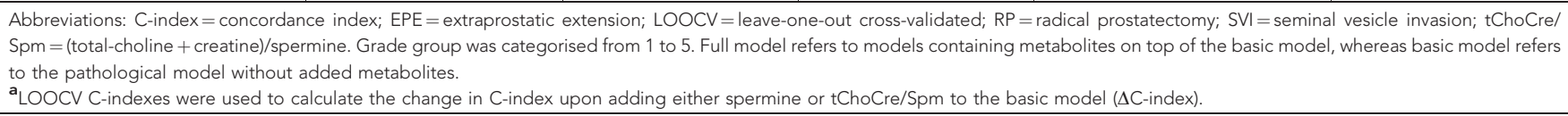 } \\
\hline
\end{tabular}

applied to dichotomise the patient populations in the two other cohorts. Kaplan-Meier analyses showed good reproducibility for spermine and citrate, and fair reproducibility for tChoCre/Cit (Supplementary Figure S6). The cutoff value for tChoCre/Spm could not significantly separate the groups in the NTNU1 and NTNU2 cohorts.

Associations of metabolites with clinicopathology. The Spermine and the citrate concentrations were negatively correlated with Grade Group and pT-stage, whereas tChoCre/Spm and tChoCre/ Cit were positively correlated with Grade Group and pT-stage (Figure 3 and Supplementary Figure S7). Using the Grade Groups from the HR-MAS-analysed tissue samples gave similar correlation coefficients as using diagnostic Grade Groups from the RP specimens (results not shown). Citrate and tChoCre/Cit had higher correlations to Grade Group than spermine and tChoCre/ Spm. Extraprostatic extension was negatively correlated with spermine and citrate concentrations, whereas a positive correlation was observed for tChoCre/Spm and tChoCre/Cit. Seminal vesicle invasion was more modestly associated with the four metabolites/ metabolite ratios, and none of the metabolites correlated with patient age at operation. Of note, patient age, RP Grade Group, and extraprostatic extension were all positively correlated.

\section{DISCUSSION}

In this study, we demonstrate by ex vivo HR-MAS MRS that both the concentration of spermine and the tChoCre/Spm ratio in radical prostatectomy specimens are independent biomarkers of recurrence. A similar trend was found for citrate concentration. A combination of metabolite concentrations and standard clinicopathological parameters gave better accuracies towards predicting recurrence than clinicopathological parameters alone, although the relative increases in predictive accuracies were modest. Furthermore, decision curve analyses revealed a net benefit of adding metabolic fingerprinting to already established risk factors, with spermine granting the greatest overall improvement in net benefit. Thus, metabolic fingerprinting may serve as an adjunct predictive parameter to established decision-making nomograms. 


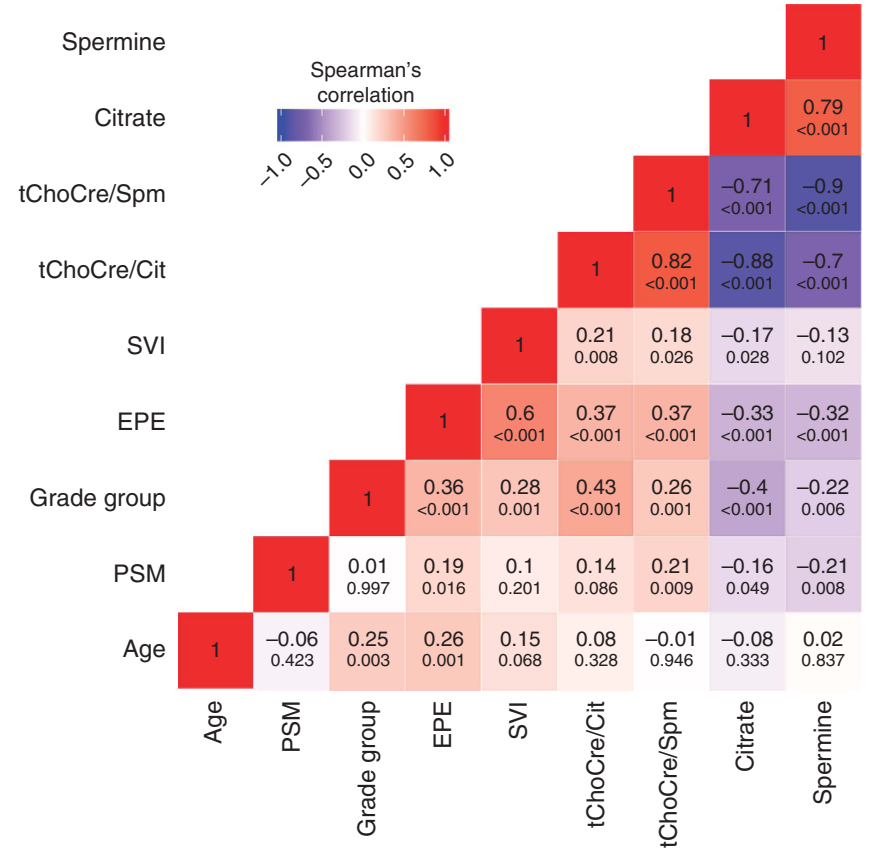

Figure 3. Correlation heatmap. Correlations between metabolites and clinicopathological factors. The Spearman's correlation coefficients are shown inside the boxes (large font), with corresponding $P$-values below. The colour scale indicates the sign of the correlation coefficients and the degree of correlation, where blue indicates negative correlation, white no correlation, and red positive correlation. EPE, extraprostatic extension; PSM, positive surgical margins; SVI, seminal vesicle invasion; tChoCre/Cit, (total-choline+creatine)/citrate; tChoCre/Spm, (totalcholine+creatine)/spermine.

Spermine and citrate concentrations were the metabolites that best correlated with recurrence within 5 years following prostatectomy. The relative levels, but not concentrations, of polyamines, therein spermine, have previously been shown to predict BCR in prostate cancer (Maxeiner et al, 2010). In support of this, a recent in vivo MRSI study demonstrated that the number of voxels with undetectable levels of polyamines was associated with recurrence (Zakian et al, 2016). Furthermore, polyamine concentrations have been reported to be reduced in malignant compared with benign prostate tissue (Van Der Graaf et al, 2000), and further decreased from low to high Gleason score (Giskeodegard et al, 2013).

Like spermine, citrate concentrations are significantly lower in tumours with high compared with low Gleason score (Giskeodegard et al, 2013), likely due to loss of capacity to accumulate zinc during neoplasia and progression leading to increased inhibition of citrate accumulation (Costello and Franklin, 1997; Bertilsson et al, 2012). Hence, both citrate and spermine are markers for benign, glandular structures, and their levels are expected to drop during dedifferentiation (Zakian et al, 2016). In support of previous reports (Swanson et al, 2003; Giskeodegard et al, 2013), we observed that spermine and citrate concentrations were significantly negatively correlated with Grade Group in radical prostatectomy specimens, although the correlation for spermine was modest. Both metabolites are constituents of the seminal fluid, and are most likely produced by luminal cells due to their positive association with luminal space (Lynch et al, 1994; Sandsmark et al, 2017). The finding that spermine, but not citrate, remains independently associated with recurrence in multivariate models, may relate to spermine's lower adherence to Gleason grade. This indicates a tissue architecture-independent association of spermine with prostate cancer aggressiveness.

Both Spermine oxidase (SMOX) and Spermidine/Spermine N1-acetyltransferase (SAT1) are enzymes that catalyse reactions in the polyamine pathway, with reactive oxygen species as byproducts (Goodwin et al, 2008; Huang et al, 2015). Interestingly, both enzymes are believed to play a role in prostate cancer progression (Goodwin et al, 2008; Huang et al, 2015). We have previously shown that when the tissue composition was balanced between prostate cancer and normal tissue samples, a significant upregulation of SAT1 and SMOX accompanied by decreased spermine levels, but without a significant upregulation of spermine synthase was measured in cancer samples (Tessem et al, 2016). This fits with a significant reduction in spermine concentration in prostate cancer samples. These observations may explain the association of spermine with prostate cancer recurrence. However, the polyamine pathway is complex and involved in multiple signalling pathways in human cells (Pegg, 2009) and whether lower spermine levels relates to dedifferentiation and loss of luminal characteristics of prostate tissue should be further investigated.

Aside from the proposed utility of postoperative ex vivo HRMAS MRS to predict recurrence, in vivo MRSI sequences may easily be added to multiparametric MR imaging (mpMRI) protocols before treatment (Shukla-Dave et al, 2009, 2012). Of the available mpMRI sequences, MRSI has primarily been applied in research settings because of its dependence on radiologist expertise on the field, prolonged overall scan time, and the associated costs (Loffroy et al, 2015). Implementation of standardised and faster MRSI protocols at higher magnetic field strengths, and computer-assisted spectral interpretation, may circumvent issues related to reproducibility of MRSI across institutions due to varied degree of radiologist training. This could, in turn, lower the overall costs of implementation of MRSI in the clinic. Importantly, studies have shown that the molecular information provided by MRSI may improve sensitivity towards detection and localisation of clinically significant prostate cancers compared with MRI alone (Wefer et al, 2000; Barentsz et al, 2012; Weinreb et al, 2016).

As the current study was performed on radical prostatectomy specimens, the interpretation of the results is limited to a postsurgery setting where treatment decision-making is limited to adjuvant or salvage treatment modalities. Ultimately, due to the large clinical challenge of improving the sensitivity towards identifying high-risk patients, as well as the specificity towards identifying truly indolent disease, the incremental value of adding MRSI protocols in the diagnostic setting should be investigated. Our study indicates that intratumoural metabolites may add value to clinically applied nomograms, and the translational potential from ex vivo HR-MAS MRS to in vivo MRSI has previously been demonstrated through a positive correlation between preoperative MRSI and HR-MAS MRS data from spatially matched tissue samples (Selnaes et al, 2013). Furthermore, the prognostic value of spermine presented in this study is in line with a previous MRSI study performed preoperatively where the authors looked at the number of polyamine-free image voxels (Zakian et al, 2016), indicating that spermine and possibly other polyamines may indeed predict risk for recurrence.

We hypothesise that MRSI may have a presurgical clinical applicability in detecting recurrent and aggressive characteristics in patients diagnosed with low- and intermediate-risk cancers. These patients may be offered radical treatment or adjuvant approaches such as radiation therapy and/or hormonal therapy (Zapatero et al, 2015), as well as extended lymph node dissection (Gordetsky and Epstein, 2016). Furthermore, mpMRI-based approaches may detect tumours not discovered by biopsies (Hambrock et al, 2010), and confirmation of low- or very-lowrisk diagnoses may aid urologists in identifying patients eligible for active surveillance. Thus, future studies should be performed to establish the clinical utility of MRSI in prostate cancer precision medicine. 
We recognise some limitations of this study. First, the cohorts collectively contain a modest number of patients, and despite the fair reproducibility observed between the cohorts, validation in larger cohorts balanced in terms of clinicopathological parameters should be conducted. Second, the retrospective design may have introduced potential confounding that we have not been able to control for.

In summary, high concentration of spermine and low tChoCre/ Spm ratio, determined by HR-MAS MRS of prostate cancer tissue, were associated with shorter time to recurrence following radical prostatectomy. Both spermine and tChoCre/Spm are visible in in vivo MRSI, which opens for clinical translation of these candidate metabolic biomarkers.

\section{ACKNOWLEDGEMENTS}

We thank the patients who agreed to supply tissue and clinical follow-up data. We also thank the Biobank 'Registry and Biobank for Urological Diseases', 'Biobank 1', and the 'MR Biobank', and the people responsible for collecting and storing these data. We would like to thank pathologist Trond Viset (St Olavs Hospital, Trondheim, Norway) for the grading of tissue in the NTNU1 and NTNU2 cohorts. Turid Follestad (NTNU, Trondheim, Norway) and Håkon Ramberg (OUS, Oslo, Norway) participated in counselling regarding statistical analyses, of which we are grateful. We acknowledge Alan J. Wright (Cancer Research UK, Cambridge, UK) for assistance with LCModel quantification of the MR spectra. We thank the Movember Foundation, the Norwegian Cancer Society, and Oslo University Hospital for funding the project. Finally, we thank Torill E. Sjøbakk (NTNU, Trondheim, Norway) and the Core Facility for MR (NTNU, Trondheim, Norway) for technical assistance and availability of experimental equipment.

\section{CONFLICT OF INTEREST}

The authors declare no conflict of interest.

\section{REFERENCES}

Barentsz JO, Richenberg J, Clements R, Choyke P, Verma S, Villeirs G, Rouviere O, Logager V, Futterer JJ. European Society of Urogenital R (2012) ESUR prostate MR guidelines 2012. Eur Radiol 22: 746-757.

Benjamini Y, Hochberg Y (1995) Controlling the false discovery rate - a practical and powerful approach to multiple testing. J R Stat Soc Ser B 57: 289-300.

Bertilsson H, Angelsen A, Viset T, Skogseth H, Tessem MB, Halgunset J (2011) A new method to provide a fresh frozen prostate slice suitable for gene expression study and MR spectroscopy. Prostate 71: 461-469.

Bertilsson H, Tessem MB, Flatberg A, Viset T, Gribbestad I, Angelsen A, Halgunset J (2012) Changes in gene transcription underlying the aberrant citrate and choline metabolism in human prostate cancer samples. Clin Cancer Res 18: 3261-3269.

Bishoff JT, Freedland SJ, Gerber L, Tennstedt P, Reid J, Welbourn W, Graefen M, Sangale Z, Tikishvili E, Park J, Younus A, Gutin A, Lanchbury JS, Sauter G, Brawer M, Stone S, Schlomm T (2014) Prognostic utility of the cell cycle progression score generated from biopsy in men treated with prostatectomy. J Urol 192: 409-414.

Budczies J, Klauschen F, Sinn BV, Gyorffy B, Schmitt WD, Darb-Esfahani S, Denkert C (2012) Cutoff Finder: a comprehensive and straightforward Web application enabling rapid biomarker cutoff optimization. PLoS One 7: e51862.

Canfield SE, Kibel AS, Kemeter MJ, Febbo PG, Lawrence HJ, Moul JW (2014) A guide for clinicians in the evaluation of emerging molecular diagnostics for newly diagnosed prostate cancer. Rev Urol 16: 172-180.

Costello LC, Franklin RB (1997) Citrate metabolism of normal and malignant prostate epithelial cells. Urology 50: 3-12.
Cullen J, Rosner IL, Brand TC, Zhang N, Tsiatis AC, Moncur J, Ali A, Chen Y, Knezevic D, Maddala T, Lawrence HJ, Febbo PG, Srivastava S, Sesterhenn IA, Mcleod DG (2015) A biopsy-based 17-gene genomic prostate score predicts recurrence after radical prostatectomy and adverse surgical pathology in a racially diverse population of men with clinically low- and intermediate-risk prostate cancer. Eur Urol 68: 123-131.

Erho N, Crisan A, Vergara IA, Mitra AP, Ghadessi M, Buerki C, Bergstralh EJ, Kollmeyer T, Fink S, Haddad Z, Zimmermann B, Sierocinski T, Ballman KV, Triche TJ, Black PC, Karnes RJ, Klee G, Davicioni E, Jenkins RB (2013) Discovery and validation of a prostate cancer genomic classifier that predicts early metastasis following radical prostatectomy. PLoS One 8: e66855.

Giskeodegard GF, Bertilsson H, Selnaes KM, Wright AJ, Bathen TF, Viset T, Halgunset J, Angelsen A, Gribbestad IS, Tessem MB (2013) Spermine and citrate as metabolic biomarkers for assessing prostate cancer aggressiveness. PLoS One 8: e62375.

Goodwin AC, Jadallah S, Toubaji A, Lecksell K, Hicks JL, Kowalski J, Bova GS, De Marzo AM, Netto GJ, Casero Jr. RA (2008) Increased spermine oxidase expression in human prostate cancer and prostatic intraepithelial neoplasia tissues. Prostate 68: 766-772.

Gordetsky J, Epstein J (2016) Grading of prostatic adenocarcinoma: current state and prognostic implications. Diagn Pathol 11: 25.

Hambrock T, Somford DM, Hoeks C, Bouwense SA, Huisman H, Yakar D, Van Oort IM, Witjes JA, Futterer JJ, Barentsz JO (2010) Magnetic resonance imaging guided prostate biopsy in men with repeat negative biopsies and increased prostate specific antigen. J Urol 183: 520-527.

Hansen AF, Sandsmark E, Rye MB, Wright AJ, Bertilsson H, Richardsen E, Viset T, Bofin AM, Angelsen A, Selnaes KM, Bathen TF, Tessem MB (2016) Presence of TMPRSS2-ERG is associated with alterations of the metabolic profile in human prostate cancer. Oncotarget 7: 42071-42085.

Harrell Jr. FE, Califf RM, Pryor DB, Lee KL, Rosati RA (1982) Evaluating the yield of medical tests. JAMA 247: 2543-2546.

Huang W, Eickhoff JC, Mehraein-Ghomi F, Church DR, Wilding G, Basu HS (2015) Expression of spermidine/spermine N(1) -acetyl transferase (SSAT) in human prostate tissues is related to prostate cancer progression and metastasis. Prostate 75: 1150-1159.

Kumar D, Gupta A, Nath K (2016) NMR-based metabolomics of prostate cancer: a protagonist in clinical diagnostics. Expert Rev Mol Diagn 16: 651-661.

Lagemaat MW, Breukels V, Vos EK, Kerr AB, Van Uden MJ, Orzada S, Bitz AK, Maas MC, Scheenen TW (2016) 1)H MR spectroscopic imaging of the prostate at 7T using spectral-spatial pulses. Magn Reson Med 75: 933-945.

Loffroy R, Chevallier O, Moulin M, Favelier S, Genson PY, Pottecher P, Crehange G, Cochet A, Cormier L (2015) Current role of multiparametric magnetic resonance imaging for prostate cancer. Quant Imag Med Surg 5 : 754-764.

Lynch MJ, Masters J, Pryor JP, Lindon JC, Spraul M, Foxall PJ, Nicholson JK (1994) Ultra high field NMR spectroscopic studies on human seminal fluid, seminal vesicle and prostatic secretions. J Pharm Biomed Anal 12: 5-19.

Maxeiner A, Adkins CB, Zhang Y, Taupitz M, Halpern EF, Mcdougal WS, Wu CL, Cheng LL (2010) Retrospective analysis of prostate cancer recurrence potential with tissue metabolomic profiles. Prostate 70: $710-717$.

Pegg AE (2009) Mammalian polyamine metabolism and function. IUBMB Life 61: $880-894$.

Pound CR, Partin AW, Eisenberger MA, Chan DW, Pearson JD, Walsh PC (1999) Natural history of progression after PSA elevation following radical prostatectomy. JAMA 281: 1591-1597.

Provencher SW (1993) Estimation of metabolite concentrations from localized in vivo proton NMR spectra. Magn Reson Med 30: 672-679.

Sandsmark E, Hansen AF, Selnaes KM, Bertilsson H, Bofin AM, Wright AJ, Viset T, Richardsen E, Drablos F, Bathen TF, Tessem MB, Rye MB (2017) A novel non-canonical Wnt signature for prostate cancer aggressiveness. Oncotarget 8: 9572-9586.

Sciarra A (2010) Words of wisdom. Re: Retrospective analysis of prostate cancer recurrence potential with tissue metabolomic profiles. Maxeiner A, Adkins CB, Zhang Y, et al. Prostate 2010;70:710-7. Eur Urol 58: 315.

Selnaes KM, Gribbestad IS, Bertilsson H, Wright A, Angelsen A, Heerschap A, Tessem MB (2013) Spatially matched in vivo and ex vivo MR metabolic profiles of prostate cancer - investigation of a correlation with Gleason score. NMR Biomed 26: 600-606. 
Shukla-Dave A, Hricak H, Akin O, Yu C, Zakian KL, Udo K, Scardino PT, Eastham J, Kattan MW (2012) Preoperative nomograms incorporating magnetic resonance imaging and spectroscopy for prediction of insignificant prostate cancer. BJU Int 109: 1315-1322.

Shukla-Dave A, Hricak H, Ishill N, Moskowitz CS, Drobnjak M, Reuter VE, Zakian KL, Scardino PT, Cordon-Cardo C (2009) Prediction of prostate cancer recurrence using magnetic resonance imaging and molecular profiles. Clin Cancer Res 15: 3842-3849.

Steinseifer IK, Van Asten JJ, Weiland E, Scheenen TW, Maas MC, Heerschap A (2015) Improved volume selective (1) H MR spectroscopic imaging of the prostate with gradient offset independent adiabaticity pulses at 3 tesla. Magn Reson Med 74: 915-924.

Swanson MG, Vigneron DB, Tabatabai ZL, Males RG, Schmitt L, Carroll PR, James JK, Hurd RE, Kurhanewicz J (2003) Proton HR-MAS spectroscopy and quantitative pathologic analysis of MRI/3D-MRSI-targeted postsurgical prostate tissues. Magn Reson Med 50: 944-954.

Tessem MB, Bertilsson H, Angelsen A, Bathen TF, Drablos F, Rye MB (2016) A balanced tissue composition reveals new metabolic and gene expression markers in prostate cancer. PLoS One 11: e0153727.

Van Der Graaf M, Schipper RG, Oosterhof GO, Schalken JA, Verhofstad AA, Heerschap A (2000) Proton MR spectroscopy of prostatic tissue focused on the detection of spermine, a possible biomarker of malignant behavior in prostate cancer. MAGMA 10: 153-159.

Vickers AJ, Elkin EB (2006) Decision curve analysis: a novel method for evaluating prediction models. Med Decis Making 26: 565-574.

Ward JF, Blute ML, Slezak J, Bergstralh EJ, Zincke H (2003) The long-term clinical impact of biochemical recurrence of prostate cancer 5 or more years after radical prostatectomy. J Urol 170: 1872-1876.

Wefer AE, Hricak H, Vigneron DB, Coakley FV, Lu Y, Wefer J, Mueller-Lisse U, Carroll PR, Kurhanewicz J (2000) Sextant localization of prostate cancer: comparison of sextant biopsy, magnetic resonance imaging and magnetic resonance spectroscopic imaging with step section histology. J Urol 164: 400-404.

Weinreb JC, Barentsz JO, Choyke PL, Cornud F, Haider MA, Macura KJ, Margolis D, Schnall MD, Shtern F, Tempany CM, Thoeny HC, Verma S (2016) PI-RADS prostate imaging - reporting and data system: 2015, version 2. Eur Urol 69: 16-40.

Weis J, Von Below C, Tolf A, Ortiz-Nieto F, Wassberg C, Haggman M, Ladjevardi S, Ahlstrom H (2016) Quantification of metabolite concentrations in benign and malignant prostate tissues using 3D proton MR spectroscopic imaging. J Magn Reson Imaging 45: $1232-1240$.

Zakian KL, Hatfield W, Aras O, Cao K, Yakar D, Goldman DA, Moskowitz CS, Shukla-Dave A, Tehrani YM, Fine S, Eastham J, Hricak H (2016) Prostate MRSI predicts outcome in radical prostatectomy patients. Magn Reson Imaging 34: 674-681.

Zapatero A, Guerrero A, Maldonado X, Alvarez A, Gonzalez San Segundo C, Cabeza Rodriguez MA, Macias V, Pedro Olive A, Casas F, Boladeras A, De Vidales CM, Vazquez, De La Torre ML, Villa S, Perez, De La Haza A, Calvo FA (2015) High-dose radiotherapy with short-term or longterm androgen deprivation in localised prostate cancer (DART01/05 GICOR): a randomised, controlled, phase 3 trial. Lancet Oncol 16: 320-327.

This work is published under the standard license to publish agreement. After 12 months the work will become freely available and the license terms will switch to a Creative Commons AttributionNonCommercial-Share Alike 4.0 Unported License.

Supplementary Information accompanies this paper on British Journal of Cancer website (http://www.nature.com/bjc) 